\title{
Caracterización de la reactividad conductual frente al manejo y su relación con el cortisol sanguíneo, contusiones y pH de la carne en novillos ${ }^{\#}$
}

\author{
Characterisation of behavioural reactivity in steers during handling and its relationship with blood \\ cortisol, bruising and meat $\mathrm{pH}$
}

\section{De Freslon ${ }^{\mathrm{a}}$, A Strappinit, M Soto-Gamboa ${ }^{\mathrm{c}}, \mathrm{C}$ Gallo $^{\mathrm{b} *}$}

\author{
aPrograma Magíster en Ciencias mención Salud Animal, Facultad de Ciencias Veterinarias, Universidad Austral de Chile, Valdivia, Chile. \\ 'Instituto de Ciencia Animal, Facultad de Ciencias Veterinarias, Universidad Austral de Chile, Valdivia, Chile. \\ 'Instituto de Ciencias Ambientales y Evolutivas, Facultad de Ciencias, Universidad Austral de Chile, Valdivia, Chile.
}

\begin{abstract}
SUMMARY
In order to explore the capacity of behavioural tests to predict the incidence of high $\mathrm{pH}$ meat (dark cutting) in commercial setting, we assessed the relationship between behavioural reactivity, physiological parameters and meat quality in steers. Fourty-two steers were weighed individually in a scale $(2.5 \times 3 \times 2.5 \mathrm{~m})$ to assess two behavioural parameters: crush test and exit velocity. Repeatability of the behavioural responses during 3 weighing events was calculated and a consistency scale was created to determine consistency of the parameters. Blood samples were collected from each steer after the second crush test and at bleeding, then analyzed for serum cortisol. Bruising and $\mathrm{pH}$ were registered after slaughter on the carcasses. Crush score was highly consistent $(\mathrm{r}=0.81-0.87)$ and exit velocity moderately consistent $(\mathrm{r}=0.44-0.60)$, however individual differences were detected in the "acclimation" and "sensitization" to the test through time. Higher crush score was associated with higher on farm cortisol concentration. Steers producing carcasses with multiple bruises (3-4) had a higher serum cortisol concentration at bleeding $(\mathrm{P}<0.05)$ than steers with less carcass bruising. Behavioural parameters, carcass bruising and meat $\mathrm{pH}$ were not statistically associated. We conclude that it is important to evaluate behavioural reactivity using serial tests and we recommend further exploration on the capacity of reactivity tests to detect differences in reactivity that can be reflected in differences in meat quality parameters.
\end{abstract}

Key words: steers, reactivity, meat $\mathrm{pH}$.

\section{RESUMEN}

Con el motivo de indagar en la predicción de la incidencia de $\mathrm{pH}$ elevado (corte oscuro) en base a pruebas conductuales aplicables en situaciones comerciales, se evaluó la relación entre la reactividad conductual y parámetros fisiológicos y de calidad de carne en novillos. Cuarenta y dos novillos

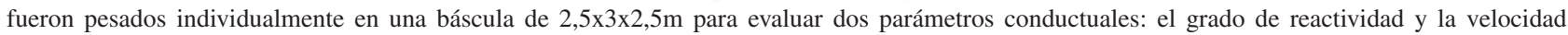
de salida. Para determinar la consistencia de las respuestas, se calculó la repetibilidad de las respuestas durante tres pesajes y se creó una escala cualitativa de consistencia. La concentración de cortisol sérico fue determinada post-pesaje en el predio y al desangrado, las contusiones y el pH fueron registrados en la canal. El grado de reactividad fue altamente repetible $(r=0,81-0,87)$ y la velocidad de salida medianamente repetible $(r=0,44-0,60)$, sin embargo, se detectaron diferencias individuales en la "habituación" y "sensibilización" a la prueba. Los animales con mayor grado de reactividad tuvieron mayores concentraciones de cortisol sérico en el predio. No hubo asociación entre las respuestas conductuales, las contusiones en la canal y el pH final de la carne. Novillos cuyas canales presentaron múltiples contusiones (3-4) tuvieron una concentración de cortisol promedio al desangrado significativamente $(\mathrm{P}<0,05)$ más alta que novillos con menos contusiones en la canal. Se concluye que la reactividad debe ser medida en forma seriada y se recomienda indagar más sobre la capacidad de las pruebas de reactividad para detectar diferencias en reactividad que puedan reflejarse en diferencias en parámetros de calidad de la carne.

Palabras clave: bovinos, reactividad, $\mathrm{pH}$ muscular.

\section{INTRODUCCIÓN}

La respuesta al estrés presenta una variabilidad considerable entre animales, no solamente en su percepción del evento o factor estresante, sino también en la coordinación

Aceptado: 07.11.2013.

\# FONDECYT 1120757

* cgallo@uach.cl de su respuesta (Moberg 2001). Tanto la percepción como la coordinación son moduladas por varios factores intrínsecos tales como la genética, el sexo, la edad y el estado fisiológico, además de la experiencia y el aprendizaje del animal (Boissy 1995). La forma conductual y fisiológica del individuo de responder a situaciones potencialmente estresantes se conoce bajo el término de reactividad al estrés (Terlouw 2005), y es una característica del temperamento (Manteca y Deag 1993). El estudio de las dife- 
rencias individuales en la respuesta al estrés relacionadas con el temperamento, permitiría identificar animales con dificultad en adaptarse a desafíos y/o cambios ambientales y predecir las respuestas conductuales (Van Reenen y col 2004).

Actualmente se sabe que el estrés puede tener efectos detrimentales en los niveles de producción, en la calidad de la carne y en el bienestar del animal (Von Borell 1995, Voisinet y col 1997). Existe un cierto patrón en la respuesta al estrés con respecto a la presentación de $\mathrm{pH}$ elevado en la carne (que lleva al DFD o corte oscuro). A partir de ello existe un interés creciente en cuantificar el impacto del estrés pre-sacrificio y explorar estrategias para mitigar las pérdidas relacionadas con el fenómeno. Sin embargo, poco se sabe sobre las causas intrínsecas (propias de cada animal) de la variabilidad entre individuos en la presentación de corte oscuro en animales del mismo tipo sometidos a las mismas condiciones presacrificio (Ferguson y Warner 2008). La caracterización de animales que tienen mayor riesgo de producir carnes oscuras, firmes y secas permitiría desarrollar estrategias de manejo para reducir o prevenir la ocurrencia de este problema. Existe cierta evidencia de que el temperamento está relacionado con indicadores fisiológicos de estrés en bovinos (King y col 2006, Miranda de La Lama y col 2013); siendo el estrés un componente determinante en la ocurrencia de $\mathrm{pH}$ elevado en la canal, se podría esperar que distintos niveles de estrés, dados por el temperamento, influyan en distinto grado en la presentación de $\mathrm{pH}$ elevado.

El objetivo de este estudio fue caracterizar la reactividad al manejo, mediante la evaluación de respuestas conductuales asociadas al temperamento, y evaluar su relación con el cortisol plasmático, el número de contusiones en las canales y el pH de la carne en novillos.

\section{MATERIAL Y MÉTODOS}

\section{ANIMALES}

Se utilizaron 42 bovinos machos castrados (novillos) de aproximadamente 28 meses de edad, alojados en un predio para engorda (Chile, $40^{\circ} 23^{\prime}$ y $72^{\circ} 59^{\prime} \mathrm{W}$ ) durante el otoño. Se seleccionó un predio que tenía históricamente un porcentaje elevado de canales con $\mathrm{pH}$ superior a 5,8 en los animales que enviaba a faena. Los animales habían sido adquiridos en distintas ferias ganaderas de la región y mantenidos juntos aproximadamente por 14 meses en el mismo predio, previo al inicio del estudio; correspondían fenotípicamente a cruzas de las razas Frisón rojo y negro, y Angus, conformando un grupo de animales representativos de las características de la producción de carne en Chile. De un lote inicial de 80 animales, se seleccionaron 42 basado en sus conformaciones morfológicas, pesos corporales y alzada, de manera de tener animales con un rango de peso corporal entre 430 y 550 kg y de alzada similar (130 a $140 \mathrm{~cm}$ ). Estos animales se mantuvieron junto al lote inicial hasta el final del estudio en una pastura cercada de 5 hectáreas, de baja producción, por lo que eran suplementados diariamente con 30 $\mathrm{kg}$ de ensilaje de ballica (con $34 \%$ de materia seca), $3 \mathrm{~kg}$ de avena, $1,5 \mathrm{~kg}$ de heno de pastura natural y $100 \mathrm{~g}$ de sal mineral por animal. La pastura tenía un bebedero de cemento de 2x0,7x0,6 m, de fácil acceso para los animales y con agua disponible ad libitum. Los animales eran suplementados dos veces al día, y no recibían otro manejo que el pesaje y aplicación de antiparasitario al ingresar al predio y posteriormente una vez al año. Los operarios encargados de los animales no estaban entrenados en manejo y bienestar animal, pero contaban con una amplia experiencia práctica en manejo de ganado bovino.

\section{PESAJE}

Todos los animales del lote fueron pesados en tres ocasiones, separadas entre sí por 9 días (repetición $1=\mathrm{R} 1$, día 0 ; repetición $2=\mathrm{R} 2$, día 9; repetición $3=\mathrm{R} 3$, día 18), de manera de poder evaluar la consistencia de las respuestas al pesaje. El primer pesaje corresponde a la segunda exposición de los animales a este manejo (la primera exposición se efectuó 14 meses antes, al ingresar al predio). El pesaje se efectúo en una báscula tipo romana (1 a $3.000 \mathrm{~kg}$ ) de 2,5 m de ancho por 3 metros de largo, con paredes de madera de 2,5 $\mathrm{m}$ de alto, que dejaban espacios libres entre sí cada $10-20 \mathrm{~cm}$. La puerta de salida de la romana se encontraba ubicada en el lado opuesto a la puerta de entrada, ambas con sistema de deslizamiento lateral manual. Los animales eran arreados tranquilamente por dos operarios a caballo (sin palos) desde la pastura hasta un corral de espera 30 min antes del pesaje, y posteriormente conducidos en grupos de 4-5 animales a un pequeño corral de conexión con la romana (figura 1). Para el pesaje, cada animal fue introducido a la romana individualmente y aleatoriamente, donde fue identificado y pesado. Posteriormente, se abría la puerta de salida para que el animal se desplazara hacia un patio de espera contiguo. Desde la entrada del animal en la báscula, hasta la salida del cuerpo completo del animal, se registró su comportamiento mediante videocámara (Panasonic NV-GS230, posicionada encima de la báscula, a 3 metros de altura). Durante este tiempo los animales no fueron sometidos a ningún otro manejo por parte de los operarios. Las videograbaciones fueron transferidas a un computador y transformadas en archivos AVI para su análisis. Debido a que el pesaje se efectuó bajo condiciones comerciales, no se pudo controlar el tiempo de permanencia en la báscula y éste dependió principalmente del tiempo requerido para identificar y pesar cada animal (entre 17 segundos y 1 minuto); como no todos los animales se mantuvieron en la báscula por el mismo tiempo, se consideró para el análisis el tiempo mínimo de permanencia, que fueron los primeros 17 segundos desde el ingreso de cada animal a la báscula. 


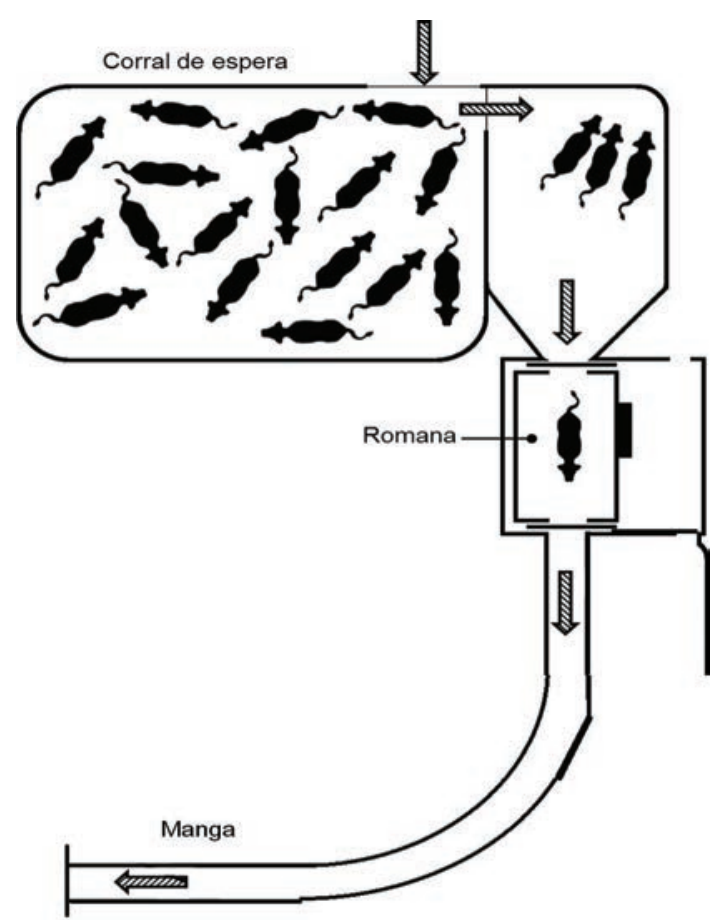

Figura 1. Plano de los corrales, romana y manga utilizados en la prueba. Las flechas indican el flujo de animales.

Plan of the pens, scale and crush used for the reactivity test. Arrows indicate the flow of the animals.

\section{GRADO DE REACTIVIDAD Y VELOCIDAD DE SALIDA}

Debido a que la báscula permitía el desplazamiento del animal, no se podía utilizar una escala de reactividad típica, como las usadas comúnmente en las pruebas de temperamento (Grandin 1993). Estas escalas de reactividad fueron desarrolladas para sistemas de báscula en brete, donde el animal no se puede desplazar, e incluso en algunos casos se encuentra inmovilizado de cuerpo entero o del cuello. Por ello se diseñó una escala bidimensional ad hoc (cuadro 1), que incorpora elementos de las escalas de reactividad en brete y de las escalas de reactividad en corral. Los grados se asignaron mediante apreciación visual del animal durante los primeros 17 segundos del pesaje, según la escala desarrollada. La velocidad de salida de cada animal fue estimada mediante una escala cualitativa apreciando el paso de cada animal, desde que éste inició el abandono de la báscula hasta su completa salida. Se le asignó a cada animal un puntaje de acuerdo a la escala propuesta por Grandin y col (1995), la cual fue adaptada a las condiciones y tipo de ganado local, que es menos exitable (cuadro 2). Los datos fueron registrados por un mismo observador (entrenado en observaciones conductuales) para eliminar una potencial fuente de variación y fueron analizados mediante el programa computacional The Observer® 5.0 (Noldus Information Technology, Wageningen, NL).
Cuadro 1. Escala cualitativa utilizada para asignar el grado de reactividad de los novillos.

Qualitative scale used to assign crush score of steers.

\begin{tabular}{lccc}
\hline & \multicolumn{3}{c}{ Estado $^{1}$} \\
\cline { 2 - 4 } Locomoción $^{2}$ & Tranquilo & Intranquilo & Alterado \\
\hline Escaso o nulo & Grado 1 & Grado 2 & Grado 3 \\
Intermedio & Grado 2 & Grado 3 & Grado 4 \\
Constante $^{4}$ & Grado 3 & Grado 4 & Grado 4 \\
\hline
\end{tabular}

${ }^{1}$ apreciación cualitativa del estado del animal; ${ }^{2}$ desplazamiento de 2 o más extremidades; ${ }^{3}$ locomoción que dura en total entre $2 / 4$ y $3 / 4$ del tiempo de registro; ${ }^{4}$ locomocion que dura en total más de $3 / 4$ del tiempo de registro.

Cuadro 2. Escala cualitativa utilizada para asignar el puntaje de velocidad de salida de los novillos (adaptado de Grandin y col 1995).

Qualitative scale used to assign exit velocity of steers (adapted from Grandin et al 1995).

\begin{tabular}{cl}
\hline Puntaje & Descripción \\
\hline 1 & Camina lento y/o alternado con paradas \\
2 & Camina continuamente a velocidad normal \\
3 & Camina a una velocidad mayor de la normal \\
4 & Trota \\
5 & Galopa \\
\hline
\end{tabular}

\section{CORTISOL PLASMÁTICO}

Después del segundo pesaje en el predio, los novillos ingresaron a una manga conectada con la salida de la báscula, donde se recolectó una muestra de sangre (3 $\mathrm{ml}$ ) por venopunción coccígea (aproximadamente $5 \mathrm{~min}$ después de la salida de cada novillo de la báscula). La primera muestra se tomó a las 10:00 h y la última muestra a las 12:30 h. Las muestras de sangre fueron etiquetadas y transportadas en hielo para posteriormente centrifugarlas por $20 \mathrm{~min}$ a $1000 \mathrm{~g}$ y almacenar el suero obtenido a $-20^{\circ} \mathrm{C}$. Las concentraciones de cortisol sérico se determinaron en duplicados de $20 \mu \mathrm{l}$ mediante radioinmunoensayo utilizando el kit comercial CORTISOL-RIA-CT® (DIAsource ImmunoAssays S.A., Bélgica; coeficiente de variación inter-ensayo 3\%).

\section{MATANZA DE LOS ANIMALES Y MEDICIONES POST- MORTEM}

Aproximadamente una semana después del último pesaje, los animales fueron enviados a una planta faenadora 
comercial. El viaje se efectuó el día anterior a la faena, en un camión con carro destinado al transporte de ganado (área total de carga disponible de $40 \mathrm{~m}^{2}$ ), a una densidad de carga de $1 \mathrm{~m}^{2}$ por $500 \mathrm{~kg}$ y duró aproximadamente cuatro horas. En la planta faenadora se respetó la conformación del grupo inicial, sin ingresar otros animales al grupo durante la espera en ayuno previo a la faena (15 horas, con acceso a agua). La matanza se efectuó en la mañana del día siguiente, a partir de las 7:00 h; los animales fueron arreados uno por uno hasta el cajón de noqueo, e insensibilizados con una pistola de proyectil retenido no penetrante, impulsado por aire comprimido. Una vez insensibilizado, cada novillo fue izado al riel de desangrado e inmediatamente al iniciarse el desangrado se recolectó la segunda muestra de sangre $(10 \mathrm{ml})$. El procesamiento de las muestras se efectúo en forma idéntica al procedimiento descrito para las muestras tomadas en el predio. También se registraron las contusiones presentes en las canales (posteriormente al eviscerado) según sus características, usando el protocolo descrito por Strappini y col (2011). Se tomó en cuenta el color, grado de severidad y número de contusiones. Solamente se registraron contusiones recientes (de menos de $24 \mathrm{~h}$ por su color rojo brillante o rojo oscuro, Strappini y col 2009), indicativas de que se produjeron en el periodo que comprende desde el arreo en el predio hasta la matanza.

Las canales se mantuvieron en una cámara de frío $\left(0-4^{\circ} \mathrm{C}\right)$ desde aproximadamente 30 min posterior al sacrificio. Se determinó el pH de las canales a las $24 \mathrm{~h}$ post-mortem, utilizando un peachímetro con electrodo de pincho introducido al centro del músculo Longissimus thoracis (entre la $9^{\mathrm{a}}$ y $10^{\mathrm{a}}$ costilla, calibrado según las recomendaciones del distribuidor y cada 5 canales).

\section{ANÁLISIS ESTADÍSTICO}

Se analizaron los resultados mediante estadística descriptiva. Como la distribución de los datos conductuales no cumplió con los supuestos de normalidad y no pudo ser satisfactoriamente transformada, se utilizó estadística no paramétrica para los análisis posteriores.

Se evaluaron cambios significativos en las respuestas conductuales entre las repeticiones 1, 2 y 3 mediante la prueba de Friedman para el grado de reactividad y la velocidad de salida. En caso de encontrarse diferencias significativas, se compararon las repeticiones pareadas mediante la prueba de contraste de Wilcoxon para muestras pareadas.

La repetibilidad conductual se evaluó mediante el coeficiente de correlación intra-clase (CCI), que representa la fracción de la variación conductual explicada por las diferencias entre individuos. El CCI se calculó con la siguiente fórmula:

$$
\mathrm{r}=\mathrm{s}_{\mathrm{A}}^{2} /\left(\mathrm{s}^{2}+\mathrm{s}_{\mathrm{A}}^{2}\right)
$$

donde $\mathrm{s}_{\mathrm{A}}^{2}$ es la varianza inter-individuos y $\mathrm{s}^{2}$ la varianza intra-individuos en el tiempo (Lessells y Boag 1987). Como uno de los intereses principales del análisis era detectar diferencias individuales en las tendencias conductuales, se crearon dos escalas cualitativas para las variables conductuales (grado de reactividad y velocidad de salida). Se denominaron 'escalas de consistencia' para efectos prácticos; cada animal recibió un puntaje de acuerdo a su respuesta en las tres repeticiones, y en base a cada escala se pudieron clasificar los animales con respecto a la consistencia de su respuesta, para complementar el análisis de los resultados obtenidos en las pruebas estadísticas (cuadro 3).

Se evaluó la asociación entre el grado de reactividad promedio y la velocidad de salida promedio mediante análisis de correlación de Spearman rho; una correlación significativa $(\mathrm{P}<0,05)$ indica que existe una relación lineal entre ambas variables.

Se evaluó la relación entre las variables conductuales (grado de reactividad promedio y velocidad de salida promedio), el cortisol sérico post-pesaje en predio y al desangrado mediante un análisis de correlación de Spearman rho. Se compararon las concentraciones de cortisol post-pesaje con las concentraciones al desangrado (prueba de Mann-Whitney).

Con respecto a las contusiones, para efectos prácticos se formaron cuatro grupos, separando animales sin contusión detectable, con 1 contusión, con 2 contusiones y con 3 o más contusiones. Se compararon los resultados de las variables en base al número de contusiones clasificado en estos cuatro grupos, con la prueba de Kruskal-Wallis. En caso de encontrarse diferencias significativas entre los tres grupos, se aplicó la prueba Mann-Whitney de contraste a posteriori.

Se efectuaron dos análisis para relacionar el $\mathrm{pH}$ con las variables conductuales: el primero con el $\mathrm{pH}$ como variable contínua, que corresponde a los valores no modificados del $\mathrm{pH}$ registrado en las canales, por lo que se utilizó la correlación de Spearman. El segundo con el pH como variable dicotómica, que corresponde a los valores de $\mathrm{pH}$ clasificados según valores inferiores a 5,8 (normales) o superiores o iguales a 5,8 (elevados) (comparación con la prueba de Mann-Whitney); esta es una de las clasificaciones más comunes que utiliza el $\mathrm{pH}=5,8$ como valor crítico para la condición de corte oscuro (Wulf y col 2002).

Todas las pruebas estadísticas de este estudio fueron calculadas con el programa computacional SPSS Statistics versión 17.0 para Windows (SPSS Inc, Chicago IL, USA). Las pruebas de diferencias y de correlación fueron de dos colas y se utilizó un nivel crítico de significancia de 0,05.

\section{RESULTADOS}

\section{CARACTERIZACIÓN DE LAS RESPUESTAS CONDUCTUALES}

Grado de reactividad. Se encontraron diferencias entre individuos reflejadas por las frecuencias expuestas en el cuadro 4. El puntaje 2 fue el más frecuentemente asignado 
Cuadro 3. Escala de consistencia a través de las tres repeticiones para evaluar el grado de reactividad y la velocidad de salida en los novillos. Consistency scale assessing crush test and exit velocity of steers through three repetitions.

\begin{tabular}{ll}
\hline Categoría & Descripción \\
\hline Constante mínimo & Animales con puntaje 1 en R1, R2 y R3 \\
Constante medio & Animales con puntaje 2 en R1, R2 y R3 \\
Constante alto & Animales con puntaje 3 en R1, R2 y R3 o puntaje 4 en R1, R2 y R3 \\
En aumento & Animales con puntaje más bajo obtenido en R1, y puntaje mas alto obtenido en R3 \\
En descenso & Animales con puntaje más alto obtenido en R1, y puntaje mas bajo obtenido en R3 \\
Inconsistente & Animales con puntaje sin tendencia a través de R1, R2 y R3 \\
\hline
\end{tabular}

$\mathrm{R} 1$ = repetición $1 ; \mathrm{R} 2$ = repetición $2 ; \mathrm{R} 3$ = repetición 3 .

Cuadro 4. Clasificación de los novillos por grado de reactividad y puntaje de velocidad de salida en las tres repeticiones (Rep). Steers classification based on crush score y exit velocity in the three repetitions (Rep).

\begin{tabular}{|c|c|c|c|c|c|c|c|c|c|}
\hline \multirow[b]{2}{*}{ Rep. } & \multicolumn{4}{|c|}{ Grado de reactividad $^{1}$} & \multicolumn{5}{|c|}{ Puntaje de velocidad de salida ${ }^{2}$} \\
\hline & $\begin{array}{c}1 \\
\%(\mathrm{n})\end{array}$ & $\begin{array}{c}2 \\
\%(n)\end{array}$ & $\begin{array}{c}3 \\
\%(n)\end{array}$ & $\begin{array}{c}4 \\
\%(\mathrm{n})\end{array}$ & $\begin{array}{c}1 \\
\%(n)\end{array}$ & $\begin{array}{c}2 \\
\%(\mathrm{n})\end{array}$ & $\begin{array}{c}3 \\
\%(n)\end{array}$ & $\begin{array}{c}4 \\
\%(n)\end{array}$ & $\begin{array}{l}5 \\
\%\end{array}$ \\
\hline 1 & $33,3(14)$ & $38,1(16)$ & $16,70(7)$ & $11,9(5)$ & $19,1(8)$ & $71,4(30)$ & $7,1(3)$ & $2,4(1)$ & 0,0 \\
\hline 2 & $23,8(10)$ & $42,9(18)$ & $19,10(8)$ & $14,3(6)$ & $21,4(9)$ & $64,3(27)$ & $14,3(6)$ & $0,0(0)$ & 0,0 \\
\hline 3 & $16,70(7)$ & $35,7(15)$ & $33,3(14)$ & $14,3(6)$ & $14,3(6)$ & $64,3(27)$ & $19,1(8)$ & $2,4(1)$ & 0,0 \\
\hline
\end{tabular}

${ }^{1}$ Referirse al cuadro $1 ;{ }^{2}$ Referirse al cuadro 2 .

(animales "intranquilos" con un desplazamiento escaso o bien animales "tranquilos" con una locomoción moderada).

Se observaron diferencias entre repeticiones (R1-R2, $\mathrm{P}<0,05$; R2-R3, P < 0,05; R1-R3, P < 0,01), con un aumento de la frecuencia de expresión de los puntajes más altos (3 y 4), y una disminución de la frecuencia para los puntajes más bajos, a medida que los animales fueron experimentando más pesajes.

La repetibilidad, calculada con el coeficiente de correlación intra-clase, fue significativa y alta (R1-R2: $\mathrm{r}=$ 0,87, $\mathrm{P}<0,01$; R2-R3: $\mathrm{r}=0,84, \mathrm{P}<0,01$; R1-R3: $\mathrm{r}=$ $0,81, \mathrm{P}<0,01)$.

La categoría más representada en la escala de consistencia fue la categoría en aumento (38,1\%; figura 2 ), que representa novillos con un grado de reactividad mayor en la repetición 3 que en la repetición 1 . La mayoría $(52,4 \%)$ de los animales mantuvo una respuesta constante al pesaje (reflejado por las categorías constante mínimo, contante medio y constante alto) y ningún animal fue clasificado dentro de la categoría en descenso.

Velocidad de salida. Se encontraron diferencias entre individuos reflejadas por las frecuencias expuestas en el cuadro 4. El puntaje más frecuentemente expresado fue el puntaje 2, que engloba animales que salieron de la romana caminando constantemente a un paso normal.
No se observaron diferencias significativas en la tendencia central de la variable entre las tres repeticiones $(\mathrm{P}>0,05)$. Al igual que el grado de reactividad, la repetibilidad de la velocidad de salida fue significativa (R1-R2: $\mathrm{r}=0,60, \mathrm{P}<0,01 ; \mathrm{R} 2-\mathrm{R} 3: \mathrm{r}=0,56, \mathrm{P}<0,01 ; \mathrm{R} 1-\mathrm{R} 3: \mathrm{r}=0,44$, $\mathrm{P}=0,01)$. La mayoría de los animales mantuvo una velocidad de salida constante a través de los tres puntajes (59,5\%; figura 2). El grado de reactividad y la velocidad de salida estuvieron moderadamente correlacionados $(\mathrm{rs}=0,36, \mathrm{P}<0,05)$.

\section{RELACIÓN ENTRE LAS RESPUESTAS CONDUCTUALES, FISIOLÓGICA Y DE CALIDAD DE CARNE}

Cortisol sérico. Con respecto al cortisol en predio (postsegundo pesaje), cinco animales tuvieron valores faltantes para esta variable por tener concentraciones inferiores al mínimo detectable de $9 \mu \mathrm{g} / \mathrm{L}$. La concentración promedio de cortisol post-pesaje en el predio fue significativamente menor que la del cortisol al desangrado (medias \pm DE respectivas: $30,89 \pm 16,46 \mu \mathrm{g} / \mathrm{L}, 64,35 \pm 24,29 \mu \mathrm{g} / \mathrm{L}$; $P<0,01)$. No hubo correlación entre la velocidad de salida y el cortisol post pesaje o al desangrado $(\mathrm{P}>0,05)$. El grado de reactividad tampoco se correlacionó con el cortisol al desangrado $(\mathrm{P}>0,05)$, sin embargo, se corre- 


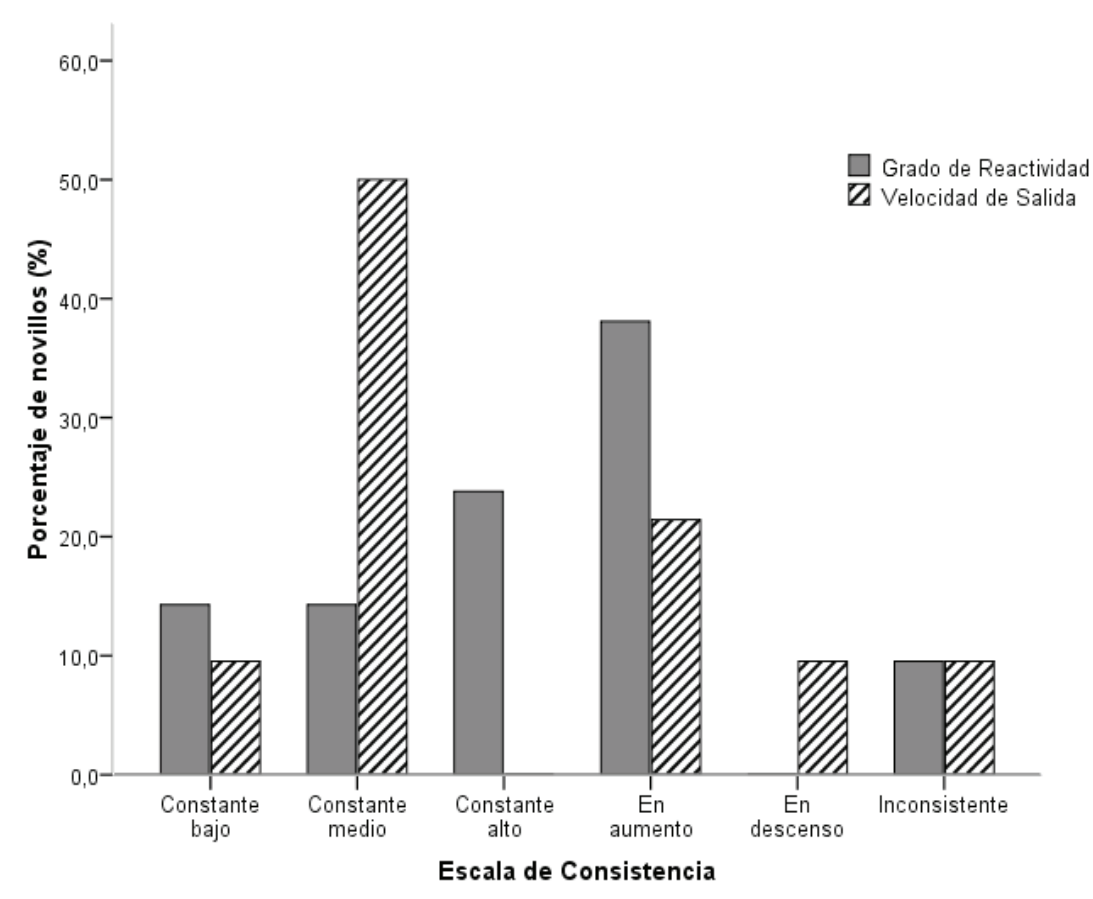

Figura 2. Porcentaje de novillos por categoría clasificados según la escala de consistencia para el grado de reactividad y velocidad de salida.

Percentage of steers classified according to the consistency scale for reactivity score and exit velocity.

lacionó con el cortisol post-pesaje en predio $(\mathrm{r}=0,34, \mathrm{P}$ $<0,05)$. Se decidió comparar las concentraciones de cortisol post-pesaje en base a los puntajes de reactividad de la primera repetición. Así, los novillos que obtuvieron un puntaje 4 de reactividad tuvieron un cortisol sanguíneo post-pesaje significativamente más alto que los animales que obtuvieron un puntaje $1(\mathrm{P}<0,01), 2(\mathrm{P}<0,05)$ o 3 $(\mathrm{P}<0,05)$ (figura 3$)$.

Contusiones. Con respecto al análisis de las contusiones en las canales, un 26,8\% de los novillos no presentó contusión, mientras que un $31,7 \%, 31,7 \%$ y $9,8 \%$ tuvieron 1 , 2 y 3 o 4 contusiones respectivamente. No se encontraron diferencias para las variables conductuales grado de reactividad y velocidad de salida al ser comparadas por el número de contusiones $(\mathrm{P}>0,05)$.

Los animales cuyas canales presentaron tres o cuatro contusiones tuvieron un cortisol promedio al desangrado significativamente más alto que los animales con canales con una o con dos contusiones (media \pm EE respectivas: $99,32 \pm 19,33 \mu \mathrm{g} / \mathrm{L} ; 58,45 \pm 4,3 \mu \mathrm{g} / \mathrm{L} ; 60,61 \pm 4,7 \mu \mathrm{g} / \mathrm{L}$; $\mathrm{P}<0,05)$.

$p H$. Los novillos tuvieron un $\mathrm{pH}_{24}$ promedio de 6,04 $\pm 0,43$, con un mínimo de 5,52 y un máximo de 7 . Un $46,3 \%(\mathrm{n}=19)$ registró un $\mathrm{pH}_{24}$ inferior a 5,8, y el 53,7\% $(\mathrm{n}=22)$ restante un $\mathrm{pH}_{24}$ superior o igual a 5,8. No se encontró correlación entre el grado de reactividad y el $\mathrm{pH}_{24}$, ni entre la velocidad de salida y el $\mathrm{pH}_{24}(\mathrm{P}>0,05)$. Tampoco se observaron diferencias significativas al comparar, en base al pH clasificado de forma dicotómica (inferior a 5,8; superior o igual a 5,8), las medianas del grado de reactividad (medianas \pm EE respectivas: $2,11 \pm 1,10,2,05$ $\pm 9,95 ; \mathrm{P}>0,05)$ y de la velocidad de salida (medianas \pm EE respectivas: $1,95 \pm 0,78,1,91 \pm 0,43$; $\mathrm{P}>0,05$ ).

No se encontró asociación significativa entre el pH de la canal, el cortisol sérico post-pesaje $(\mathrm{P}>0,05)$ y al desangrado $(\mathrm{P}>0,05)$. Al comparar los resultados del cortisol sérico en base al $\mathrm{pH}$ clasificado de forma dicotómica (inferior a 5,8; superior o igual a 5,8), tampoco se observaron diferencias significativas en las medias de aquellas variables $(\mathrm{P}>0,05)$.

\section{DISCUSIÓN}

Si bien la mayoría de los novillos fueron calificados con grado de reactividad intermedio (estado tranquilo a intranquilo y con un grado de desplazamiento nulo a intermedio) y abandonaron la báscula a una velocidad lenta a normal, se observó un aumento general en el grado de reactividad hacia los pesajes siguientes. Ello indica que los animales tendieron a desplazarse más y/o fueron más inquietos a medida que experimentaron más pesajes, reflejando un aumento general en la excitabilidad. Sin embargo, el grado de reactividad fue altamente repetible a través del tiempo, y la velocidad de salida tuvo una re- 


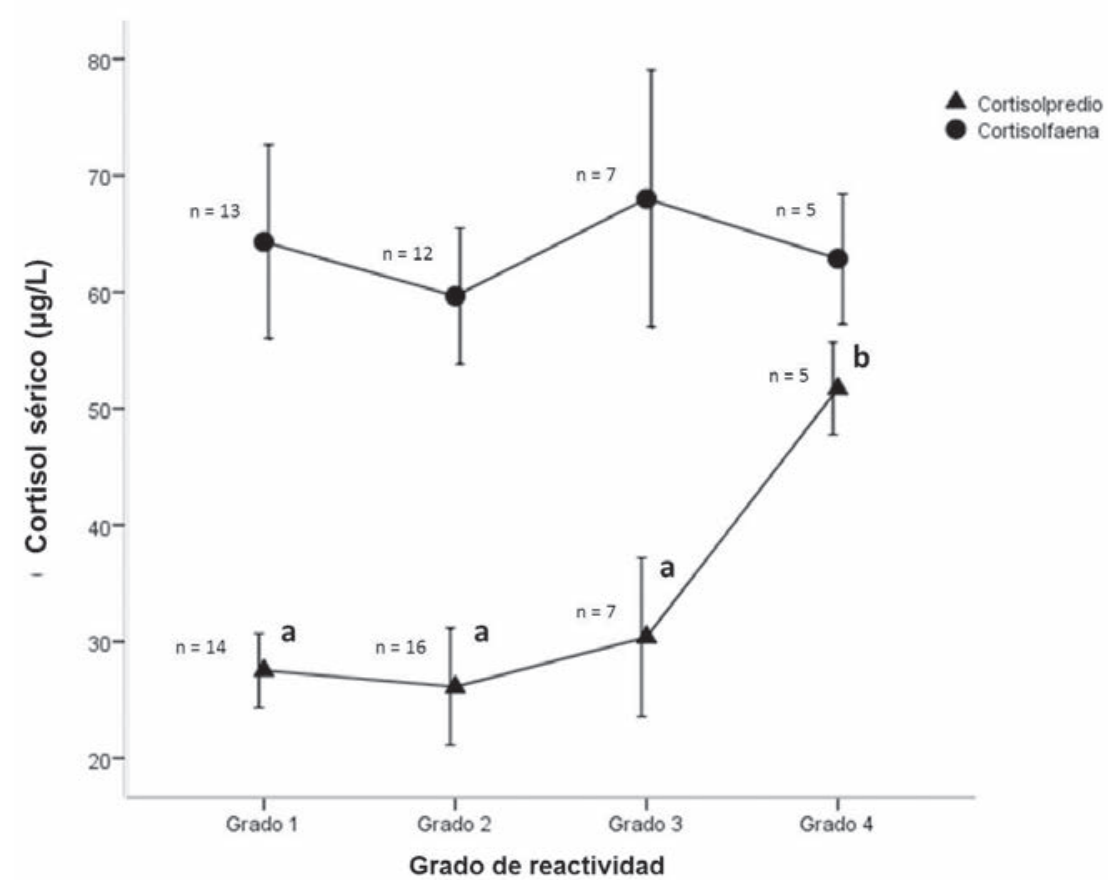

Figura 3. Medias $(\boldsymbol{\mu g} / \mathrm{L})+$ EE de las concentraciones séricas de cortisol en novillos en predio post-pesaje (cortisolpredio) y al desangrado (cortisolfaena) según grado de reactividad de los animales. Letras diferentes indican diferencias significativas $(\mathrm{P}<0,05)$.

On farm post-weighing (cortisolpredio) and at slaughter (cortisolfaena) serum cortisol means $(\mu \mathrm{g} / \mathrm{L})+$ EE classified according to crush score. Significant differences are marked with different letters $(\mathrm{P}<0.05)$.

petibilidad menor, que según el criterio de Bruton y col (2000), no cumple con el coeficiente mínimo de 0,6 para una repetibilidad aceptable.

Para ser una característica del temperamento, la respuesta conductual debe ser relativamente constante en el tiempo para cada individuo (Burrow 1997). Si bien la repetibilidad evalúa la consistencia individual a través del tiempo, la compara además con la variabilidad entre los individuos del grupo y por ende es una característica de la población más que información sobre individuos. Es decir, cuando el coeficiente de repetibilidad es significativamente alto, no significa necesariamente que todos los individuos de la población son consistentes en su respuesta (Bell y col 2009). Al repetir el experimento, existe un proceso de adaptación, donde los animales recolectan y procesan información, y adaptan su conducta consecuentemente (Erhard y Mendl 1999). A raíz de ello, es probable que algunos animales se hayan "habituado" al pesaje más rápidamente que otros, y que consecuentemente la intensidad de su respuesta haya disminuido (Martin y Réale 2008). A su vez, posiblemente otros animales al contrario se hayan "sensibilizado", aumentando la magnitud de su respuesta ante el mismo evento (van Oers y col 2005). Por ende, la variabilidad en la capacidad y velocidad de "habituación" o "sensibilizacion" afecta la repetibilidad de las mediciones (Hayes y Jenkins 1997) y también constituye una variabilidad indivi- dual en cuanto a la repetibilidad (Erhard y Mendl 1999). Ello se vio evidenciado en los resultados de la escala de consistencia, donde se observa que un poco más de un tercio de los animales aumentó su respuesta al pesaje en el tiempo. Por lo tanto, si bien la mayoría de los animales mantuvo una respuesta constante al pesaje, un porcentaje considerable de animales $(38,1 \%$; figura 2$)$ aumentó su puntaje a través de los pesajes. Estos resultados indican que existe un porcentaje de animales que varían su respuesta a medida que van experimentando más pesajes, mientras que otros se mantienen constantes en su respuesta, aun cuando la repetibilidad calculada en base a la muestra es significativa y elevada. Por otro lado, la mayoría de los animales mantuvo una velocidad de salida constante a través de los tres puntajes, sin embargo la repetibilidad de esta variable fue baja. El hecho de que la mayoría de los novillos obtuvo el mismo puntaje de velocidad de salida probablemente tuvo un impacto en el coeficiente de correlación intra-clase, que depende de la variabilidad entre individuos.

Con respecto a los valores de cortisol sanguíneo postpesaje, fueron más elevados que los valores registrados en novillos en reposo por Oyarce y col (2002) (media \pm DE: $1,4 \pm 1,18 \mu \mathrm{g} / \mathrm{dL}$ ) y similares a los registrados por Alvarez y col (2002) en novillos en manga (tubo). Era de esperar encontrar que todos los animales tuvieran un cortisol más elevado en el desangrado que en el predio 
(post-pesaje) debido al estrés acumulado del periodo pre-sacrificio (Voisinet y col 1997, Tadich y col 2002). Ello explicaría la falta de diferencia significativa en el cortisol al desangrado, entre grupos de diferente grado de reactividad. En cuanto al cortisol post-pesaje, el hecho de que novillos con un puntaje 4 de reactividad tuvieron un cortisol sanguíneo post-pesaje significativamente más alto que los animales con puntajes menores, indica posiblemente que para animales que reaccionaron con mayor reactividad conductual, el pesaje fue un evento más estresante que para el resto de los animales. Es ampliamente reconocido que las respuestas neuroendocrinas son dependientes de la forma en la cual un animal percibe la situación (von Borell 1995). No obstante, los niveles de cortisol pueden ser afectados por muchos factores, $\mathrm{y}$ debido a ello la respuesta fisiológica no siempre varía en conjunto con la respuesta conductual.

Los animales cuyas canales presentaron tres o cuatro contusiones tuvieron una concentración de cortisol promedio al desangrado significativamente más alta que animales con canales con una o dos contusiones. Debido a que no hubo relación entre cortisol post-pesaje en predio y contusiones en la canal, y a la ausencia de relación entre las contusiones y las variables conductuales, es posible que los animales con tres o cuatro contusiones hayan tenido un cortisol al desangrado superior por un efecto relacionado con los eventos que causaron las contusiones o por el dolor mismo, más que por la reactividad.

Se ha postulado que el estrés pre-sacrificio lleva a la disminución de las reservas de glucógeno del músculo, lo cual puede a su vez llevar a una escasa formación de ácido láctico post-mortem y con ello a un $\mathrm{pH}$ muscular elevado (Warriss 1990). Era de esperar entonces que el $\mathrm{pH}$ de la canal de los animales más estresables, en este caso los más reactivos y que presentaron concentraciones de cortisol más elevadas, hubiese sido superior al $\mathrm{pH}$ de la canal de animales menos estresables y viceversa. Sin embargo, en este estudio no se encontró relación entre el $\mathrm{pH}$ de la canal y las variables conductuales. Es posible que el alto porcentaje de animales con canales con $\mathrm{pH}$ superior a 5,8 haya enmascarado asociaciones entre las variables. La presencia de canales con $\mathrm{pH}$ elevado es frecuente en las plantas faenadoras de Chile y se han reportado prevalencias de corte oscuro entre un $4 \%$ a $10 \%$, pudiendo incluso alcanzar a $40 \%$ en algunos grupos (Gallo 2003). Los resultados concuerdan con el hecho de que se seleccionó un predio con alta prevalencia de $\mathrm{pH}$ elevado.

Varios estudios publicados hasta la fecha han fallado en demostrar una asociación entre la respuesta conductual de los animales en el predio y el $\mathrm{pH}$ final de la carne (Voisinet y col 1997, Boissy y col 2000, Petherick y col 2002, Barbosa y col 2006, King y col 2006). Tampoco se ha encontrado asociación entre el manejo pre-sacrificio y el pH final; animales que sufrieron un periodo pre-sacrificio intencionalmente estresante (manejo adicional, aislamiento social y ejercicio) no tuvieron valores de $\mathrm{pH}_{24}$ su- perior a animales que tuvieron un periodo pre-sacrificio con presencia limitada de factores estresantes (Bourguet y col 2010). Amtmann y col (2006) tampoco encontraron diferencias significativas en cuanto a las concentraciones de cortisol (tomado en manga en el predio antes del transporte) entre animales que posteriormente presentaron canales con $\mathrm{pH}_{24}<5,8$ y $\mathrm{pH}_{24} \geq 5,8$. Todo ello indica que la relación entre las variables conductuales, cortisol y $\mathrm{pH}$ de la canal puede no ser directa como se ha postulado, y que varios factores no se suelen tomar en cuenta, como por ejemplo los factores metabólicos post-mortem involucrados. Los determinantes de la calidad final de la carne son complejos y multifactoriales (Ferguson y Warner 2008), y es posible que además de factores detrimentales para la calidad de la carne, existan factores compensatorios, resultando en un $\mathrm{pH}$ final difícil de anticipar, sobre todo si no se toma en cuenta esa amplia gama de factores.

En conclusión, bajo las condiciones del presente estudio, las diferencias individuales de los novillos en las respuestas conductuales observadas en el tiempo, demuestran que una sola medición no es suficiente para predecir la reacción de los novillos en el tiempo. Por otro lado, el estudio evidenció que es necesario indagar más sobre la capacidad de adaptación de los animales al pesaje como factor predictor del cortisol sanguíneo y de calidad de la carne. Debido a que en el presente estudio no se encontró relación entre la reactividad, las contusiones y el pH de la carne, creemos necesario investigar más sobre la capacidad de las pruebas de reactividad de predecir las respuestas de los animales que redundan en una menor calidad de la carne, bajo condiciones comerciales.

\section{REFERENCIAS}

Alvarez E, N Tadich, C Gallo. 2002. Efecto de diferentes métodos de arreo sobre algunas variables sanguíneas indicadoras de estrés en bovinos. Resúmenes XII Congreso de Medicina Veterinaria, Chillán, Chile.

Amtmann V, C Gallo, G van Schaik, N Tadich. 2006. Relaciones entre el manejo antemortem, variables sanguíneas indicadoras de estrés y $\mathrm{pH}$ de la canal en novillos. Arch Med Vet 38, 259-264.

Barbosa I, V Fischer, G Dorneles. 2006. Relação entre o genótipo e o temperamento de novilhos em pastejo e seu efeito na qualidade da carne. $R$ Bras Zootec 35, 519-526.

Bell A, S Hankison, K Laskowski. 2009. The repeatability of behaviour: a meta-analysis. Anim Behav 77, 771-783.

Boissy A. 1995. Fear and fearfulness in animals. Q Rev Biol 70, 165-191.

Boissy A, G Trillat, X Fernandez, X Boivin, J Sapa, J Culioli, P Le Neindre. 2000. Réactivité comportementale et indicateurs de qualité de la viande chez le taurillon. Rencontres autour des Recherches sur les Ruminants 7, 58-61.

Bourguet C, V Deiss, M Gobert, D Durand, A Boissy, EMC Terlouw. 2010. Characterising the emotional reactivity of cows to understand and predict their stress reactions to the slaughter procedure. Appl Anim Behav Sci 125, 9-21.

Bruton A, JH Conway, ST Holgate. 2000. Reliability: What is it 
and how is it measured? Physiotherapy 86, 94-99.

Burrow HM. 1997. Measurements of temperament and their relationships with performance traits of beef cattle: A review. Anim Breed Abstr 65, 477-495.

Erhard HW, M Mendl. 1999. Tonic immobility and emergence time in pigs - more evidence for behavioural strategies. Appl Anim Behav Sci 61, 227-237.

Ferguson DM, RD Warner. 2008. Have we underestimated the impact of pre-slaughter stress on meat quality in ruminants? Meat Sci 80, 12-19.

Gallo C. 2003. Efectos de diferentes condiciones de manejo previo al faenamiento sobre la calidad de la carne en bovinos. Revista Intercampo 6, 8-12.

Grandin T. 1993. Behavioral agitation during handling of cattle is persistent over time. Appl Anim Behav Sci 36, 1-9.

Grandin T, M Deesing, J Struthers, A Swinker. 1995. Cattle with hair whorl patterns above the eyes are more behaviorally agitated during restraint. Appl Anim Behav Sci 46, 117-123.

Hayes JP, SH Jenkins. 1997. Individual variation in mammals. J Mammal 78, 274-293.

King DA, CE Schuehle Pfeiffer, RD Randel, TH Welsh Jr., RA Oliphint, BE Baird, KO Curley Jr., RC Vann, DS Hale, JW Savell. 2006. Influence of animal temperament and stress responsiveness on the carcass quality and beef tenderness of feedlot cattle. Meat Sci 74, 546-556.

Lessells CM, PT Boag. 1987. Unrepeatable repeatabilities: a common mistake. Auk 104, 116-121.

Manteca X, JM Deag. 1993. Use of physiological measures to assess individual differences in reactivity. Appl Anim Behav Sci 37, 265-270.

Martin JG, D Réale. 2008. Temperament, risk assessment and habituation to novelty in eastern chipmunks, Tamias striatus. Anim Behav 75, 309-318.

Miranda de la Lama GC, M Pascual-Alonso, A Guerrero, P Alberti, S Alierta, P Sans, JP Gajan, M Villarroel, A Dalmau, A Velarde, MM Campo, F Galindo, MP Santolaria, C Sañudo, GA María. 2013. Influence of social dominance on welfare, production and the quality of meat from young beef bulls. Meat Sci 94, 432-437.

Moberg GP. 2001. Biological response to stress, implications to animal welfare. In: Moberg GP, Mench JA (eds). The biology of animal stress - Basic principles and implications for animal welfare. CABI Publishing, Oxon, UK, Pp 1-22.
Oyarce J, N Tadich, C Gallo. 2002. Determinación de algunos constituyentes sanguíneos indicadores de estrés, en novillos en reposo. Resúmenes de la XXVII Reunión Anual de la Sociedad Chilena de Producción Animal, Chillán, Chile.

Petherick J, RG Holroyd, VJ Doogan, BK Venus. 2002. Productivity, carcass and meat quality of lot-fed Bos indicus cross steers grouped according to temperament. Aust $J$ Exp Agric 42, 389-398.

Strappini AC, JHM Metz, C Gallo, B Kemp. 2009. Origin and assessment of bruises in beef cattle at slaughter. Animal 3, 728-736.

Strappini AC, K Frankena, JHM Metz, C Gallo, B Kemp. 2011. Characteristics of bruises in carcasses of cows sourced from farms or from livestock markets. Animal 6, 502-509.

Tadich N, C Gallo, T Knowles, A Aranis. 2002. Concentración de algunas variables sanguíneas indicadoras de estrés antes y durante la sangría en novillos. Resúmenes del XII Congreso de Medicina Veterinaria, Chillán, Chile.

Terlouw C. 2005. Stress reactions at slaughter and meat quality in pigs: genetic background and prior experience. Livest Prod Sci 94, 125-135.

Van Oers K, G de Jong, AJ van Noordwijk, B Kempenaers, PJ Drent. 2005. Contribution of genetics to the study of animal personalities: a review of case studies. Behaviour 142, 1185-1206.

Van Reenen CG, B Engel, LFM Ruis-Heutinck, JTN Van der Werf, WG Buist, RB Jones, HJ Blockhuis. 2004. Behavioural reactivity of heifer calves in potentially alarming test situations: a multivariate and correctional analysis. Appl Anim Behav Sci 85, 11-30.

Voisinet BD, T Grandin, SF O'Connor, JD Tatum, MJ Deesing. 1997. Bos indicus-cross feedlot cattle with excitable temperaments have tougher meat and a higher incidence of borderline dark cutters. Meat Sci 46, 367-377.

Von Borell E. 1995. Neuroendocrine integration of stress and significance of stress for the performance of farm animals. Appl Anim Behav Sci 44, 219-227.

Warriss PD. 1990. The handling of cattle pre-slaughter and its effects on carcass and meat quality. Appl Anim Behav Sci 28, 171-186.

Wulf DM, RS Emnett, JM Leheska, SJ Moeller. 2002. Relationships among glycolytic potential, dark cutting (dark, firm, and dry) beef, and cooked beef palatability. J Anim Sci 80, 1895-1903. 
